\title{
Simulación de eventos discretos de la cadena logística de exportación de commodities
}

\author{
Discrete event simulation of the commodities' supply chain exportation
}

\author{
María Alejandra Guerrero Hernández $\quad$ André Felipe Henriques Librantz ${ }^{1}$ \\ Recibido 5 de noviembre de 2012, aceptado 6 de noviembre de 2013 \\ Received: November 5, 2012 Accepted: November 6, 2013
}

\begin{abstract}
RESUMEN
El presente trabajo utiliza la técnica de simulación de eventos discretos como metodología de apoyo a la decisión en un problema complejo con múltiples variables; el objetivo es modelar las operaciones logísticas involucradas en la exportación marítima de commodities en las presentaciones de carga suelta y granel desde doce empresas productoras hasta el puerto marítimo. La simulación permite analizar la sensibilidad de los principales parámetros y variables del sistema, con el fin de definir diferentes modelos de planeación de despachos. Los escenarios muestran que es posible obtener una reducción de los inventarios y de los costos de mantenimiento, así como también mejorar los indicadores de servicio y como consecuencia aumentar la tasa de utilización de los recursos.
\end{abstract}

Palabras clave: Simulación de eventos discretos, puertos marítimos, ProModel, exportación marítima.

\begin{abstract}
This paper uses the technique of discrete event simulation as methodology for decision support in a complex problem with multiple variables; the objective is to model the logistics operations involved in the maritime exportation of commodities in the presentations of bulk and bags from twelve producers until the seaport. The simulation allows analyzing the sensitivity of the key parameters and system variables in order to define different models of planning offices. Results showed that it is possible to reduce the inventory and maintenance costs, as well as improving the service performance indicators and consequently increasing the resources utilization rate.
\end{abstract}

Keywords: Discrete events simulation, seaport, ProModel, maritime exportation.

\section{INTRODUCCIÓN}

El modelado de sistemas reales cuyo desempeño depende en gran medida de parámetros aleatorios, como la demanda o el tiempo de servicio, es hoy un amplio campo de investigación, tanto para los desarrollos teóricos como para las aplicaciones. Un modelo de simulación es un modelo descriptivo de un proceso o sistema, que usualmente incluye parámetros para representar diferentes configuraciones del sistema o proceso.
El uso de modelos de simulación puede reemplazar la realización de experimentos en sistemas reales y aquellos proyectos que aún se encuentran en fase de desarrollo [1], permitiendo experimentar, evaluar y comparar muchos sistemas alternativos [2].

Para modelar y simular un sistema real es necesario implementar un modelo matemático aplicado a la simulación, pueden ser utilizados lenguajes de programación como C++, Visual Basic, Pascal; o programas de simulación como por ejemplo, ProModel, Arena, Extend y SLAM [3].

\footnotetext{
1 Universidad Nove de Julho (UNINOVE). Avenida Francisco Matarazzo No 612. Código Postal: 05001-001. São Paulo, Brasil. E-mail: malejandragh@gmail.com; librantz@uninove.br
} 
ProModel es un software de simulación de eventos discretos altamente flexible, que permite la interacción con otras herramientas computacionales como Excel, y su integración con Excel lo vuelve altamente flexible y amigable [4].

Los escenarios o estrategias definidos usando la simulación permiten obtener un análisis de sensibilidad de las diferentes variables, al alterar todos o algunos de los parámetros de entrada al sistema, permite obtener medidas de desempeño que reflejen la calidad del servicio prestado y la sensibilidad de los indicadores ante las variaciones del sistema. La técnica de elaboración de escenarios es un tema que ha sido tratado por varios autores,

La simulación es una metodología que se ha popularizado en los últimos años, diversos procesos logísticos, productivos y de servicios han sido mejorados con la ayuda de la simulación y el modelaje matemático [5-10].

\section{METODOLOGÍA}

El sistema modelado corresponde a los procesos desarrollados por un operador logístico, que tiene como objetivo planear, programar y realizar las operaciones logísticas de exportación de commodities en las presentaciones de carga suelta y granel, por vía marítima, para 12 empresas del sector. La carga suelta consiste en bultos sueltos o individuales, manipulados y embarcados como unidades separadas, paquetes, sacos, cajas, tambores, piezas y el producto a granel es una carga constituida por sólidos que vienen sin empaque o envase y no pierden su condición en ninguna de las distintas fases de la operación portuaria.

El operador logístico realiza la programación de los pedidos y coordina junto con las 12 empresas proveedoras la salida de los camiones desde sus instalaciones hasta el puerto marítimo, así mismo se encarga de todos los procesos administrativos y operativos desarrollados dentro del puerto marítimo.

Este operador cuenta con instalaciones alquiladas dentro del puerto marítimo, 3 bodegas, la primera de granel, con capacidad para almacenar 40.000 toneladas, y la segunda y tercera para carga suelta, con capacidad de almacenar 7.140 estibas, conjuntamente. La operación logística tanto para la exportación de carga suelta y granel incluye los siguientes procesos:

1. Programación de los despachos desde las empresas proveedoras. 2. Embarque del producto en camiones. 3. Transporte por vía terrestre. 4. Entrada de los camiones al puerto marítimo y realización de las documentaciones necesarias. 5. Control de calidad. 6. Almacenaje. 7. Carga del producto en la motonave como carga suelta, granel y contenedores (es importante resaltar que en el presente trabajo solo serán abordadas las operaciones de carga suelta y granel). El cargue de los sacos y el granel en las motonaves se realiza separadamente y con equipo especializado diferente.

Uno de los principales problemas para este operador logístico es la planeación de las operaciones y la sincronización del envío de los camiones desde las 12 empresas proveedoras. La falta de sincronización ha generado operaciones poco eficientes que se reducen en dos problemas principales y opuestos:

- Salida de los camiones de las empresas proveedoras con muchos días de anticipación antes del atraque de la motonave que irán a atender, lo que desemboca en altos niveles de inventario dentro de las bodegas alquiladas dentro del puerto marítimo.

- Falta de carga en puerto cuando se encuentra la motonave atracada y los camiones aún no han llegado con el producto. Estos dos problemas son usuales, y generan altos costos para la empresa, para el primer caso, el costo asociado corresponde al mantenimiento del inventario, y el segundo problema acarrea penalizaciones, el operador logístico debe pagar a la naviera una multa por cada día de demora en el proceso de carga del producto.

Por su parte, la eficiencia en las operaciones es premiada con el pago de compensaciones, el operador logístico recibe estos premios a base de la reducción de días de cargue con relación a lo planeado.

\section{PROCEDIMIENTOS}

Debido a la naturaleza no determinística que presenta el proceso sujeto a análisis, además de su estructura dinámica y compleja, se hace recomendable el modelaje de la situación problema mediante 
herramientas de simulación de eventos discretos. Este esquema permite representar la naturaleza estocástica del sistema y simplificar la representación de todas las variables y elementos que a él pertenecen. Para esto fue seleccionado el software de simulación de eventos discretos ProModel, por su alta flexibilidad para el modelaje de procesos.

Como estrategia, el análisis de las operaciones se realizó basada en la información correspondiente a un mes característico de la operación, mes en el cual fueron atendidas 5 motonaves con 62.400 toneladas de producto, considerada una muestra adecuada, de acuerdo con la información estudiada, la cantidad de motonaves atendidas por mes es estable, por lo tanto, esta muestra garantiza que cualquier estacionalidad en el proceso estará incluida.

Para las variables de entrada del modelo de simulación, la representatividad de los resultados se comprobó con pruebas de bondad de ajuste, Chi-Cuadrado, usando el software Stat Fit, paquete de análisis estadísticos que acompaña ProModel.

Para todos los casos, las pruebas fueron realizadas considerando una confianza de $95 \%, \alpha=0,05$.

El ajuste fue comprobado con el factor P-value. La hipótesis nula considera que los datos se ajustan a las distribuciones calculadas.

Así, la hipótesis nula es aceptada si P-value $>\alpha$, de lo contrario se rechaza y se asume que los datos no se ajustan a la distribución calculada.

Los indicadores representados como variables discretas fueron usados de esta forma debido a la falta de datos históricos para su análisis estadístico.

A continuación se presentan las variables, sus distribuciones y el resultado del cálculo de p-value.

- Tiempo de transporte de los camiones desde cada una de las empresas proveedoras resultado de las variables distancia (desde los proveedores hasta el puerto) y velocidad promedio de los camiones $(40 \mathrm{~km} / \mathrm{h})$.

- Tiempo de movimiento de las estibas con carga suelta hasta el equipo de carga de la motonave: 1,57 minutos/estiba.

- Tiempo de movimiento de la carga a granel hasta las bandas transportadoras que desembocan en la motonave: 2,36 minutos para cada 34 toneladas.

- Tiempo de análisis de laboratorio: N(7,98; 1,97 ) minutos. $\mathrm{P}$-value $=0.12$.

- Tiempo de pesaje en el puerto $\sim \mathrm{N}(2 ; 1)$ minutos. $\mathrm{P}$-value $=0,062$.

- Tiempo segundo pesaje en el puerto $\sim \mathrm{N}(2 ; 1)$ minutos. P-value $=0,16$.

- Tiempo de descargue de producto para camiones de granel $\sim \mathrm{N}(19,51 ; 6,81)$ minutos, $\mathrm{P}$-value $=0,21$.

- Tiempo de descargue de producto para camiones de carga suelta $\sim \mathrm{N}(52,721 ; 9,030)$ minutos. $\mathrm{P}$-value $=0,089$.

\section{Situación Actual}

La información de toneladas movidas y de premios ganados para el mes analizado es presentada en la Tabla 1.

A partir de esta información se buscó mejorar los resultados con el establecimiento de nuevos posibles escenarios y la definición de parámetros que permitan dar inicio a la simulación. Los indicadores fueron analizados de manera independiente para las operaciones de carga suelta y granel.

\section{Indicadores Analizados}

- Fila media en la entrada al puerto marítimo (F)

- Tiempo promedio de un camión en el sistema - Desde que sale de la empresa proveedora hasta que sale del puerto - (TS).

- Premios ganados por reducción de días de embarque para carga suelta (Premios_CS).

Tabla 1. Motonaves atendidas el mes de noviembre.

\begin{tabular}{|c|c|c|c|c|}
\hline 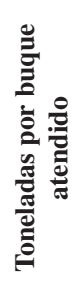 & 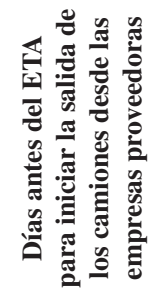 & 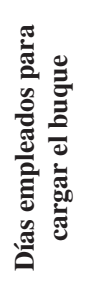 & 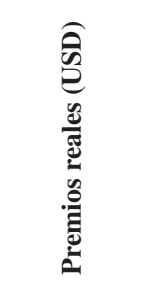 & \\
\hline 15,50 & 7,40 & 9,80 & $\$ 23.575,00$ & \multirow{4}{*}{$\begin{array}{l}\text { CARGA } \\
\text { SUELTA }\end{array}$} \\
\hline 3,50 & 6,90 & 6,10 & $\$ 1.012,00$ & \\
\hline 14,00 & 7,50 & 10,30 & $\$ 12.845,00$ & \\
\hline 4,40 & 7,60 & 2,80 & $\$ 13.255,00$ & \\
\hline \multirow[t]{2}{*}{25,00} & 6,30 & 1,50 & $\$ 23.750,00$ & GRANEL \\
\hline & \multicolumn{4}{|c|}{ TOTAL PREMIOS $\$ 74.437,00$ USD } \\
\hline
\end{tabular}


- Premios ganados por reducción de días de embarque para grane (Premios_GR).

- Media de camiones descargados por día para carga suelta (Prom_Cam/día CS).

- \# Máximo de camiones descargados por día para carga suelta (Max_Cam/día CS).

- Inventario promedio de carga suelta -Estibas-(Prom_Inv_CS).

- Máximo inventario de carga suelta -Estibas- (Max_Inv_CS).

- Media de camiones descargados por día: granel (Prom_Cam/día GR).

- \# Máximo de camiones descargados por día para granel (Max_Cam/día GR).

- Inventario promedio de granel -Toneladas-(Prom_Inv_GR).

- Máximo inventario de carga suelta -Toneladas-(Max_Inv_GR).

\section{RESULTADOS}

\section{Estrategia de Definición de Escenarios}

En el marco de la discusión sobre la disminución de costos y el incremento de beneficios en las operaciones portuarias de exportación de commodities se definió que la variable más relevante y que genera un mayor impacto en las operaciones es el número de días antes de la llegada del buque, variable que llamaremos ETA (estimated time arrival), variable que define el número de días en que los camiones deberían iniciar su salida desde las empresas proveedoras después de la fecha estimada de llegada de una motonave.

La planeación de las motonaves no puede realizarse de manera aislada y las estrategias seleccionadas deben apuntar a que el producto fluya, aun con recursos limitados y compartidos, por tanto, los escenarios simulados deben considerar ajustar y encontrar el mejor balance que produzca el mayor beneficio al estudiar estas dos alternativas:

1. En caso de que los camiones salgan desde los proveedores muchos días antes del ETA de la motonave, el nivel de inventarios acumulado el día de la llegada efectiva del barco es muy alto, causando sobrecostos por inventarios excesivos. Sin embargo, el haber mucho inventario en las bodegas genera la posibilidad de que los problemas por "falta de carga" sean mínimos, incrementando los beneficios por premios.
2. Si los camiones salen desde las empresas proveedoras con la mínima cantidad de días antes del ETA, acumularía menos inventarios permitiendo una disminución de los costos de mantenimiento de inventarios, lo que aumentaría la posibilidad de que exista falta de carga en el momento de la llegada del barco, perdiéndose la posibilidad de ganar mejores premios o teniendo incluso penalidades.

\section{Escenarios Simulados}

Situación Actual - Carga suelta: 7 días antes del ETA; granel: 15 días antes del ETA.

Escenario 1 - Carga suelta: 6 días antes del ETA; granel: 14 días antes del ETA.

Escenario 2 - Carga suelta: 5 días antes del ETA; granel: 13 días antes del ETA.

Escenario 3 - Carga suelta: para buques entre 10.000 y 15.000 toneladas, 5 días antes del ETA, para buques entre 5.000 y 10.000 toneladas 8 días antes del ETA, para buques $<5000$ toneladas, 10 días antes del ETA.

Granel: para buques $>10.000$ toneladas, $40 \%$ de la carga en puerto antes del ETA, para buques entre 5.000 y 10.000 toneladas, $50 \%$ de la carga en puerto antes del ETA, para buques $<5.000$ toneladas, $50 \%$ de la carga en puerto antes del ETA.

La Tabla 2 presenta los resultados de los escenarios simulados, permitiendo una comparación de cada una de las variables definidas frente a la modificación del parámetro de entrada días antes del ETA.

Carga suelta, al analizar estas operaciones, en el escenario actual se observa que el inventario promedio fue $28 \%$ de la capacidad de las bodegas, sin embargo, se presenta un pico máximo de inventarios que en cierto momento sobrepasa la capacidad instalada de almacenamiento dentro de las bodegas del puerto (7.140 estibas), requiriéndose el uso de bodegas alternativas.

Todos los escenarios presentan una mejora en los resultados de la empresa, los escenarios en 1 y 2 permitieron disminuir el inventario máximo de sacos llegando hasta 6.715 estibas, obteniéndose un aplanamiento de la curva de inventarios, sin afectar los premios de las motonaves de sacos.

Este beneficio también se observa en el escenario 3, en donde la planeación de las motonaves con reglas 
por diferenciación de tamaño de la motonave permite reducir el inventario máximo en las bodegas de carga suelta para 5.991 estibas, lo que hace pensar en la posibilidad de prescindir de una de las bodegas, representando un ahorro importante e impactando directamente en los costos fijos de la compañía.

Tabla 2. Cuadro comparativo de los escenarios simulados.

\begin{tabular}{|c|c|c|c|c|}
\hline Indicador & $\begin{array}{c}\begin{array}{c}\text { Situación } \\
\text { actual }\end{array} \\
\text { act }\end{array}$ & $\begin{array}{c}\text { Escenario } \\
1\end{array}$ & $\begin{array}{c}\text { Escenario } \\
2\end{array}$ & $\begin{array}{c}\text { Escenario } \\
3\end{array}$ \\
\hline $\begin{array}{l}\text { Fila media de } \\
\text { camiones en } \\
\text { la entrada del } \\
\text { puerto }\end{array}$ & 28 & 32 & 34 & 8 \\
\hline $\begin{array}{l}\text { Tiempo } \\
\text { promedio de } \\
\text { un camión } \\
\text { en el sistema } \\
\text { (horas) }\end{array}$ & 26 & 28 & 29 & 13 \\
\hline $\begin{array}{l}\text { Premios_CS } \\
\text { (\$USD) }\end{array}$ & 50.697 & 50.490 & 48.400 & 55.894 \\
\hline $\begin{array}{l}\text { Premios_GR } \\
\text { (\$USD) }\end{array}$ & 23.750 & 20.238 & 15.407 & 14.598 \\
\hline $\begin{array}{l}\text { Número } \\
\text { promedio de } \\
\text { camiones /día } \\
\text { carga suelta }\end{array}$ & 43 & 39 & 35 & 41 \\
\hline $\begin{array}{l}\text { Máximo } \\
\text { número de } \\
\text { camiones /día } \\
\text { carga suelta }\end{array}$ & 72 & 71 & 71 & 72 \\
\hline $\begin{array}{l}\text { Inventario } \\
\text { promedio } \\
\text { carga suelta } \\
\text { (estibas) }\end{array}$ & 2.044 & 1.808 & 1.572 & 1.598 \\
\hline $\begin{array}{l}\text { Inventario } \\
\text { máximo } \\
\text { carga suelta } \\
\text { (estibas) }\end{array}$ & 7.421 & 6.715 & 6.715 & 5.991 \\
\hline \begin{tabular}{|l|} 
Número \\
promedio de \\
camiones /día \\
granel \\
\end{tabular} & 46 & 46 & 46 & 46 \\
\hline $\begin{array}{l}\text { Máximo } \\
\text { número de } \\
\text { camiones /día } \\
\text { granel }\end{array}$ & 60 & 59 & 61 & 62 \\
\hline $\begin{array}{l}\text { Inventario } \\
\text { promedio } \\
\text { granel } \\
\text { (toneladas) }\end{array}$ & 3.332 & 2.856 & 2.488 & 1.800 \\
\hline $\begin{array}{l}\text { Inventario } \\
\text { máximo } \\
\text { granel } \\
\text { (toneladas) }\end{array}$ & 23.358 & 23.358 & 21.896 & 19.830 \\
\hline
\end{tabular}

Granel, en el escenario actual, el máximo inventario obtenido fue de 25.000 toneladas (capacidad de la bodega $=40.000$ toneladas), y el inventario promedio de 3.332 toneladas, presentándose una baja utilización de la bodega para este fin.

En el escenario 1, al disminuir de 15 para 14 el número de días antes del ETA, el nivel de inventario promedio disminuyó (en 884 toneladas), al igual que los premios (US\$ 3.512 por mes). Para estas operaciones se observa la alta sensibilidad de los costos ante la planeación de despachos; al no presentarse mejoras en ninguno de los escenarios propuestos, se hace pertinente que el análisis de las operaciones de granel sea realizada de manera independiente para cada nueva motonave programada y mediante la utilización de un modelo de simulación, que permita tener cuantificada la sensibilidad del sistema ante cambios en las variables.

\section{CONCLUSIONES}

Según el análisis de los diferentes escenarios desarrollados con la simulación, puede concluirse que:

La utilización de herramientas de simulación permite el análisis de sistemas complejos y de las variables implicadas en el proceso, ya que permite la investigación de la sensibilidad e impacto de los diferentes parámetros en el resultado del proceso.

Para el caso de la carga libre, puede encontrarse la alta sensibilidad del sistema a las modificaciones de fechas de envío de producto desde las empresas productoras, obteniéndose una reducción en los niveles de inventarios que podrían permitir eliminar una bodega y así reducir los costos fijos del proceso. Igualmente, la sensibilidad de este parámetro permite también que se incremente la ganancia de premios obtenidos por cumplimento en las fechas y reducción de problemas de falta de carga.

Para el caso de granel, el análisis no es tan sencillo, debido al volumen de los pedidos y la alta cantidad de camiones que deben ser movilizados para cumplir con los encargos; cada navío es un nuevo caso y debe ser analizado como un sistema independiente, es allí que la herramienta de simulación como apoyo a la planeación es útil para ayudar a la empresa a mejorar la planeación de sus operaciones por medio de una programación asertiva y flexible que permita 
minimizar el costo del inventario y maximizar los premios.

Futuros trabajos pueden desarrollarse uniendo la simulación a alguna técnica de optimización que permita encontrar nuevos resultados y evaluar diferentes escenarios para tomar mejores decisiones de forma rápida, efectiva y al menor costo.

\section{AGRADECIMIENTOS}

Los autores agradecen a la Universidad Nove de Julho (UNINOVE) por el apoyo financiero.

\section{REFERENCIAS}

[1] M.A. Law. "How to build valid and credible simulation models". Proceedings of the 38th Winter Simulation Conference. Vol. 38, pp. 58-66. 2006.

[2] J.S.I.I. Carson. "Introduction to modelling and simulation". Actas de 2003 Winter Simulation Conference. Vol. 1, pp. 7-13. New Orleans, USA. 2003.

[3] R. Shannon. "Introduction to the art and science of simulation". Proceedings of the 1998 Winter Simulation Conference, pp. 7-14. 1998.

[4] S. Hasan and M. Al-Hussein. "Advanced simulation of tower crane operation utilizing system dynamics modeling and lean principles". Proceedings of the 2010 Winter Simulation Conference. Johansson, S. Jain, J. Montoya-Torres, J. Hugan and E. Yücesan, eds. 2010

[5] M.C. Vélez Gallego, D.A. Valencia Ramírez y C.A. Castro Zuluaga. "Heurístico de simulación-optimización para la configuración de un sistema de estantería selectiva simple". Ingeniare. Revista chilena de ingeniería. Vol. $20 \mathrm{~N}^{\circ} 1$, pp. 17-24. Abril de 2012. ISSN: 0718-3305. DOI: $10.4067 /$ S0718-33052012000100003.

[6] M.J. Contreras, F. Bastleri and L. Maldonado. "Optimization of the harbor operations using simulation and surface response methodology". Revista Ingeniería Industrial. Vol. $9 \mathrm{~N}^{\circ}$ 2, pp.73-92. 2010. ISSN: 0717-9103.

[7] O. Yalcinkaya and G. Bayhan. "Modelling and optimization of average travel time for a metro line by simulation and response surface methodology". European Journal of Operational Research. Vol. 196, pp. 225233. Julio de 2009. ISSN: 0377-2217. DOI: 10.1016/j.ejor.2008.03. 010.

[8] E.M. Rubio, M.A. Sebastian y A. Sanz. "Simulación de Sistemas Flexibles de Fabricación Mediante Modelos de Realidad Virtual". Información Tecnológica. Vol. 15 $\mathrm{N}^{\circ}$ 1, pp. 49-54. 2004. ISSN: 0718-0764 DOI: $10.4067 / \mathrm{S} 0718-07642004000100008$.

[9] A. Higgins, G. Beashel and A. Harrison. "Scheduling of brand production and shipping within a sugar supply chain". Journal of the Operational Research Society. Vol. 57, pp. 490-498. June, 2005. ISSN: 0160-5682. DOI: 10.1057/palgrave.jors. 2602025.

[10] J.L. Calderón y F.C. Lario. "Simulación de Cadenas de Suministro: Nuevas Aplicaciones y Áreas de Desarrollo". Revista Información Tecnológica. Vol. 18 No 1, pp. 137-146. 2007. ISSN: 0718-0764. DOI: $10.4067 /$ S0718-07642007000100018. 